\title{
Co-Occurrence of Depression and Delinquency in Personality Types
}

\author{
JOYCE AKSE $^{1 *}$, BILL HALE ${ }^{1}$, RUTGER ENGELS $^{2}$, QUINTEN RAAIJMAKERS ${ }^{1}$ \\ and WIM MEEUS ${ }^{1}$ \\ ${ }^{1}$ Utrecht University, Adolescent Development, Utrecht, The Netherlands \\ ${ }^{2}$ Radboud University Nijmegen, Pedagogical and Educational Science, The Netherlands
}

\begin{abstract}
Although the co-occurrence between adolescent depression and delinquency has been well-studied, the nature of the longitudinal associations is not yet clear. To clarify this we examined whether personality type is a moderator in the longitudinal co-occurrence of depression and delinquency. A total of 338 young and middle adolescents completed questionnaires about depression, delinquency and personality in 3 yearly waves of the CONflict And Management Of RElationships (CONAMORE). We found that the stable overcontrollers showed the highest mean level on depression and that the stable undercontrollers showed the highest mean level on delinquency. Furthermore, we demonstrated that the longitudinal co-occurrence between depression and delinquency was best described by means of a stability model, in which personality type membership proved to be an important moderator. The three personality types differed significantly on the rank-order stability of both depression and delinquency. Copyright (C) 2006 John Wiley \& Sons, Ltd.
\end{abstract}

Key words: co-occurrence; depression; delinquency; person-centred approach; personality prototypes

\section{INTRODUCTION}

Co-occurrence is often used to describe the occurrence of two or more problem behaviours at the same point of time (Angold, Costello, \& Erkanli, 1999). Co-occurrence is often used interchangeably with comorbidity, although both terms actually refer to two separate phenomenon. The term co-occurrence refers to the identification of two or more psychopathological conditions in an individual (Keiley, Lofthouse, Bates, Dodge, \& Pettit, 2003), whereas the term comorbidity is used to define valid co-existence of two or more categorically defined and distinct disorders, such as in DSM-IV (American Psychological

*Correspondence to: Joyce Akse, Utrecht University, Adolescent Development, Utrecht, The Netherlands.

E-mail: J.Akse@fss.uu.nl 
Association, 1994). In the present study, we will investigate the co-occurrence of adolescent depression (internalising problem behaviour) and delinquency (externalising problem behaviour).

Internalising and externalising problem behaviours co-occur more often than chance rates (Krueger, Caspi, \& Moffitt, 2000) and frequently imply greater impairment, poorer responses to treatment and worse outcome over time (Keiley et al., 2003; Youngstrom, Findling, \& Calabrese, 2003), which makes it an important research topic. Although the co-occurrence between adolescent depression and delinquency has been widely studied (e.g. Angold et al., 1999; Ben-Amos, 1992; Meller \& Borchardt, 1996), the nature of the longitudinal associations is not yet clear (Beyers \& Loeber, 2003). Several theories on the mechanisms of the co-occurrence of adolescent problem behaviours have been proposed. Two of these theories suggest that one problem behaviour constitutes a risk factor for the other; however, they differ in the manner in which internalising and externalising problem behaviours predict each other. The 'failure' theory holds that externalising problems predict internalising problems; disruptive behaviour may result in rejection and a lack of support by important others, which leads to worries, anxiety and depression (Burke, Loeber, Lahey, \& Rathouz, 2005; Capaldi, 1992). For example, Burke et al. (2005) found that conduct disorder symptoms predicted subsequent depression symptoms, whereas the number of depression symptoms were not predictive of subsequent conduct disorder symptoms in adolescent boys. These results supported a failure model, whereby lack of skill and noxious behaviour lead to pervasive failures and vulnerability to depressed mood. On the other hand, the 'acting out' theory claims that internalising problems predict externalising problems; underlying depressive feelings are acted out by displaying externalising problem behaviour (Carlson \& Cantwell, 1980; Gold, Mattlin, \& Osgood, 1989). It was pointed out that experiencing depressive feelings was frequently accompanied by other problems, especially 'acting out' behaviours. This led to the concept of masked depression, in which the accompanying behaviours were thought to dominate or mask those behaviours that were associated with mood disturbances (Ben-Amos, 1992).

In addition to these two theories, another theory has been proposed to explain the cooccurrence of depression and delinquency. The stability perspective states that the cooccurrence of internalising and externalising problem behaviours is caused by non-specific risk factors, such as family history of offending, parent-child relationships or life events (Fergusson, Lynskey, \& Horwood, 1996), which lead to separate but associated problem behaviours (Krueger, 1999; Krueger, Caspi, Moffitt, \& Silva, 1998; Overbeek, Vollebergh, Meeus, Engels, \& Luijpers, 2001). This implies that there are no uni-directional or bidirectional relationships between the problem behaviours over time.

The results of the previous studies have been inconsistent with regard to the nature of the co-occurrence between depression and delinquency during adolescence (Beyers \& Loeber, 2003; Wiesner, 2003). For example, while one study found that the failure model applied to boys and that both the failure model and acting out model applied to girls (Wiesner, 2003), other studies have found that the stability model best applies to both genders (Krueger, 1999; Krueger et al., 1998; Overbeek et al., 2001). Obviously, it is not clear which of the models can best describe the co-occurrence between depression and delinquency for adolescents.

Possibly, these aforementioned studies miss a potential key factor underlying the cooccurrence between depression and delinquency, namely a person's personality (Wiesner, 2003). Indeed, personality traits have been shown to account directly for patterns of cooccurrence; neuroticism, for example, accounted for a substantial percentage of the cooccurrence between internalising and externalising problem behaviours (Krueger \& 
Markon, 2006). However, it was also noted that the patterning of individual differences in personality or the configurations of personality traits could have unique relevance to understanding the patterning of psychopathology (Krueger, 2005; Krueger et al., 2000). Therefore, we will try to clarify the co-occurrence between depression and delinquency by testing whether different co-occurrence models are valid for different personality types.

In personality research two major approaches can be distinguished. The variable-centred approach focuses on differences among individuals on a given personality trait or dimension, whereas the person-centred approach focuses on the patterning and organisation of traits within a person. Although both approaches add important insights into the understanding of personality, the present research replicates and extends recent work on personality types (e.g. Dubas, Gerris, Janssens, \& Vermulst, 2002; Hart, Burock, London, Atkins, \& Bonilla-Santiago, 2005; Robins, John, Caspi, Moffitt, \& StouthamerLoeber, 1996; van Leeuwen, Mervielde, Braet, \& Bosmans, 2004), especially the moderating role of personality types in the co-occurrence of depression and delinquency.

We will use the personality typology of Block and Block (1980) to describe the adolescents' personality, since a series of studies have shown systematic differences in depression and delinquency between the three personality types that Block and Block distinguish (Akse et al., 2004; Asendorpf \& van Aken, 1999; Dubas et al., 2002; Hart et al., 1997; Robins et al., 1996). Block and Block describe personality in terms of two continuous dimensions: ego-control and ego-resiliency. Ego-control refers to the tendency to contain emotional and motivational impulses versus the tendency to express them (overcontrol vs. undercontrol), whereas ego-resiliency refers to the tendency to respond flexibly rather than to rigidly changing situational demands, particularly stressful situations.

Robins et al. (1996) studied the personality typology of Block and Block and found egoresiliency to have an inverted U-shaped relation with ego-control, on the basis of which Robins et al. identified three personality types: resilients, overcontrollers and undercontrollers. Resilients reflected a high level of ego-resiliency and a medium level of egocontrol; overcontrollers and undercontrollers both reflected a low level of ego-resiliency; however, they differed markedly on ego-control: high and low, respectively. Additionally, Robins et al. (1996) found that these personality types had a specific profile (for more information, see Robins et al., 1996) on the dimensions of the Big Five traits (Digman, 1990; McCrae \& Costa, 1995). Consequently, Dubas et al. (2002) investigated whether the personality types could be constructed directly on the basis of the Big Five dimensions: this appeared to be possible. The personality types of Block and Block (1980) have been replicated in many studies using different informants, different methods and different statistical techniques (see: Asendorpf \& van Aken, 1999).

A number of studies have demonstrated differences in psychosocial functioning of adolescents endorsing the three personality types. Overcontrollers appeared to be more prone to higher levels of internalising problems, undercontrollers were found to be more prone to higher levels of externalising problems, whereas resilients exhibited the best psychosocial adjustment (Akse et al., 2004; Dubas et al., 2002; Hart et al., 2005; Robins et al., 1996; van Aken \& Dubas, 2004). Since these personality types show different vulnerabilities to internalising and externalising problem behaviours, and taking into account the suggestion made by Wiesner (2003) to complement the study of longitudinal co-occurrence between depression and delinquency with person-oriented approaches, it seems fruitful to investigate whether different co-occurrence models are valid for different personality types.

In light of the aforementioned, we formulated two research questions. The first research question examines the validity of the three stable personality groups. In line with earlier 


\section{J. Akse et al.}

cross-sectional studies (e.g. Akse et al., 2004; Dubas et al., 2002; Robins et al., 1996), we examined whether the stable overcontrollers have the highest mean level of depression over time, whether the stable undercontrollers have the highest mean level of delinquency over time and whether the stable resilients exhibit the lowest levels of both depression and delinquency over time, indicating the best psychosocial adjustment.

The second research question investigates whether the longitudinal co-occurrence of depression and delinquency is present during adolescence and whether personality is a moderator in the longitudinal co-occurrence between depression and delinquency. Since the co-occurrence of depression and delinquency was present in other adolescent samples in general, we expect to find it in this study as well (Overbeek et al., 2001). Furthermore, as was mentioned earlier, the exact longitudinal associations are not yet clear (e.g. Beyers \& Loeber, 2003; Wiesner, 2003) and personality types may moderate the longitudinal association between these internalising and externalising problem behaviours (Wiesner, 2003). We formulated two competing hypotheses to answer this question. Hypothesis 2a was derived from the typical pattern of depression and delinquency of the personality types. Since overcontrollers were prone to internalising problem behaviours (e.g. Robins et al., 1996), it is possible that they develop delinquency through acting out their depressed mood. Furthermore, since undercontrollers were prone to externalising problem behaviours (e.g. Robins et al., 1996), it is possible that they develop depression through failure experiences. Therefore, our hypothesis 2 a states that co-occurrence in overcontrollers will be best described by an acting out model, whereas the co-occurrence in undercontrollers will be best described by a failure model. In contrast to hypothesis $2 a$, hypothesis $2 b$ was derived from the stability perspective, which states that the co-occurrence of internalising and externalising problem behaviours is caused by non-specific risk factors. Since the personality types differ in the amount of non-specific risk factors (Hart, Atkins, \& Fegley, 2003), we assume that the co-occurrence of depression and delinquency is different for the three personality types and that this difference is stable over time. Since it is known that ego-resiliency is related to stability in the overall environment (Asendorpf \& van Aken, 1991), we hypothesize that the stability of depression as well as delinquency, including the co-occurrence between depression and delinquency, is higher for resilients than for overcontrollers and undercontrollers.

\section{METHOD}

\section{Participants}

Participants in this study were drawn from the CONflict And Management Of RElationships study (CONAMORE), which is an ongoing longitudinal study of Dutch adolescents that examines their relationships with parents and peers, as well as their emotional and behavioural states (Meeus et al., 2002). For this study, we used the first three waves of CONAMORE, collected yearly from 2001 onwards. The participating adolescents were students from 12 participating high schools located in the province of Utrecht, The Netherlands. The questionnaires about depression and delinquency and the personality questionnaire were filled in by 1088 adolescents in the first wave. The attrition was extremely low, $0.9 \%$ from wave 1 to wave 2 and $4 \%$ from wave 2 to wave 3 . 
Before we started the administration of the questionnaires, both students and their parents received written information describing the aims of the study, and if the students decided to participate, they were required to provide written informed consent. Less than $1 \%$ elected not to participate. Written informed consent from the head masters of the participating schools was obtained. The administration was performed in the homeroom study period, during which the students could fill out the questionnaires anonymously. The research assistants, who attended the administration, gave verbal instructions about the questionnaires and a written instruction was included. The research assistants collected the completed questionnaires and conducted the data entry to ensure that the data remained anonymous. Students who were absent on the day of testing were invited for a second administration or received the questionnaire by regular mail.

\section{Measures}

\section{Depression}

The Children's Depression Inventory (CDI) is a self-report questionnaire, which is used as a screen for (subclinical) depressive symptomatology in children and adolescents (Kovacs, 1985). This scale has demonstrated convergent and discriminant validity, good internal consistency and adequate test-retest reliability in previous studies (e.g. Craighead, Smucker, Craighead, \& Ilardi, 1998; Hodges, 1990). The CDI consists of 27 items; sample questions include 'I' $m$ sad all the time', 'It will never end right for me' and 'I do everything wrong'. The items were scored on a 3-point scale, ranging from 'false', 'a bit true' to 'very true'. The internal consistency of the CDI was 0.93 in wave $1,0.89$ in wave 2 and 0.90 in wave 3 .

\section{Delinquency}

The delinquency questionnaire is a self-report questionnaire, which measures the frequency of several minor offences (Baerveldt, van Rossem, \& Vermande, 2003). The use of self-report data is widespread in criminology, and it is a valid instrument when restricted to petty crime (Baerveldt, 2000). Adolescents were asked how many times they had committed 16 minor offences, such as being caught by the police for doing something, stealing a bike and deliberately damaging or breaking something in the street, in the past 12 months. The items were scored on a 4-point scale, ranging from 'never', 'once', 'two to three times' to 'four times or more'. The internal consistency of the delinquency questionnaire was 0.90 in wave $1,0.84$ in wave 2 and 0.85 in wave 3.

\section{Personality}

The personality dimensions Extraversion, Agreeableness, Conscientiousness, Emotional Stability and Openness to Experience were measured using the 30-item Big Five questionnaire (Gerris et al., 1998; Goldberg, 1992). This questionnaire has a good reliability and construct validity (e.g. Smith \& Snell, 1996).

We used the k-means clustering procedure to construct the three personality types on the basis of the Big Five dimensions (Akse et al., 2004; Dubas et al., 2002) at the three waves (Figure 1a-c ). This clustering procedure computes a mean for every individual and assigns the individual's profile to one of the three clusters on the basis of the correspondence between the cluster centre and the individual's mean. The means within a cluster should correspond highly with each other, whereas the means between the clusters must differ highly from each other. In the three waves we selected only those adolescents who scored within two standard deviations of the cluster centre (Dubas, personal communication, 12 
(a) T1 Personality

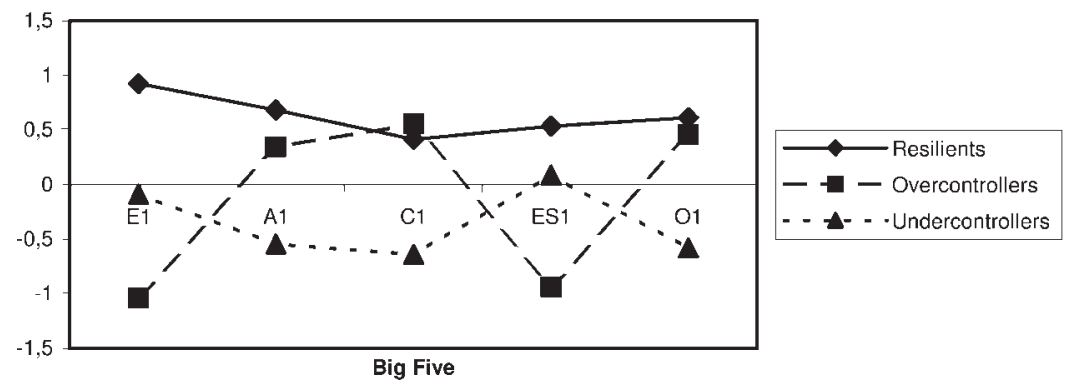

(b)

T2 Personality

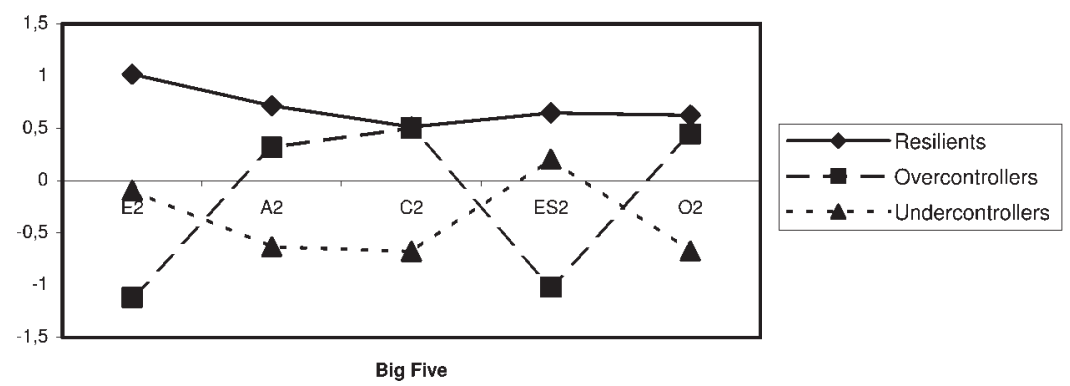

(c)

T3 Personality

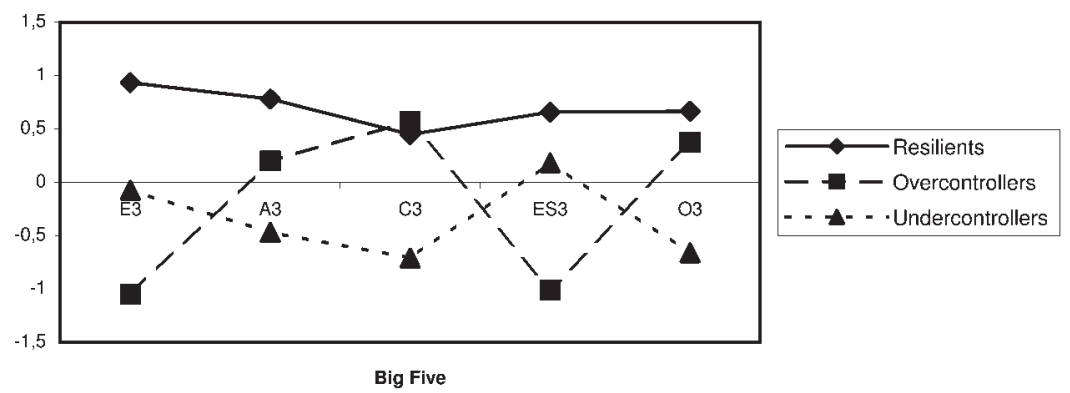

Figure 1. (a) Big Five personality profiles of the personality types in T1 (Resilients: $N=114$; Overcontrollers: $N=111$; Undercontrollers: $N=113$ ). E1: Extraversion T1; A1: Agreeableness T1; C1: Conscientiousness T1; ES: Emotional stability T1; O1: Openness to experience T1. (b) Big Five personality profiles of the personality types in T2 (Resilients: $N=114$; Overcontrollers: $N=111$; Undercontrollers: $N=113$ ). E2: Extraversion T2; A2: Agreeableness T2; C2: Conscientiousness T2; ES: Emotional stability T2; O2: Openness to experience T2. (c) Big Five personality profiles of the personality types in T3 (Resilients: $N=114$; Overcontrollers: $N=111$; Undercontrollers: $N=113$ ). E3: Extraversion T3; A3: Agreeableness T3; C3: Conscientiousness T3; ES: Emotional stability T3; O3: Openness to experience T3.

July 2004). Based on the findings of previous research in which three personality types were repeatedly found, we set the cluster number to three. Prior to the first set of cluster analyses, all dimensions scores were converted to $z$-scores. For the initial cluster centres we used a priori cluster centres derived from previous work on personality types (van Aken \& 
Dubas, 2000). More specifically, initial cluster centres for resilients were set at 0.5 on all Big Five dimensions. For the overcontrollers, the initial cluster centres were 0.5 for conscientiousness and agreeableness, and -0.5 for extraversion, emotional stability and openness. Finally, the initial cluster centres for undercontrollers were -0.5 for conscientiousness, agreeableness and openness, 0 for emotional stability and 0.5 for extraversion (Dubas et al., 2002). ${ }^{1}$ Following Dubas et al. (2002), we checked the replicability of the personality types by dividing each of the three samples randomly in two subsamples, re-running the cluster analyses for each subsample and calculating the degree of correspondence of individuals being assessed to clusters of the total sample and of the subsamples. The kappa coefficients (Cohen, 1960) for the replication samples in each wave were excellent: 0.94 and 0.95 in wave $1,0.92$ and 0.95 in wave 2 and 0.96 and 0.97 in wave 3 .

On the basis of the Big Five dimensions, we found three clusters that resembled the three personality types emerging in previous studies (e.g. Dubas et al., 2002; Robins et al., 1996). The resilient type in our study was characterised as being high on all five dimensions, as was found in other studies. Furthermore, the overcontroller type scored high on agreeableness, conscientiousness and openness and low on extraversion and emotional stability. Finally, the undercontroller type was characterised as mediocre on extraversion, agreeableness and emotional stability and as low on conscientiousness and openness. Although the undercontrollers are generally characterised as being low on agreeableness, we found them to be mediocre on this dimension. However, since they scored significantly lower on agreeableness than the other two clusters, we concluded that we replicated the three personality types in our sample (Figure 1a-c).

Of the 1088 adolescents in the first wave, 338 adolescents judged their personality type consistently over all three waves. Although it would have been interesting to investigate adolescents who judged their personality types differently over the waves as well, ${ }^{2}$ we

\footnotetext{
${ }^{1}$ During the review process of this paper, Asendorpf (2006) and Herzberg and Roth (2006) published a paper in which they suggested other initial cluster centres. They suggest that the initial cluster centres for resilients should be set at 0.5 for extraversion, conscientiousness and emotional stability and 0.25 for agreeableness and openness. For the overcontrollers, the initial cluster centres should be -1 for extraversion, 0 for agreeableness and conscientiousness, -0.5 for emotional stability and -0.25 for openness. Finally, the initial cluster centres for undercontrollers should be 0 for extraversion and openness, -0.5 for agreeableness, -1.0 for conscientiousness and -0.25 for emotional stability. We reran the k-means cluster analyses using these initial cluster centres. We compared our cluster solution with the cluster solution in which the new initial cluster centres were used and found a correspondence of 0.98 (Cohen's kappa) for the stable personality groups. When selecting only those adolescents who scored within two standard deviations of the cluster centre, Cohen's kappa increased to 1.00. Since the correspondence between the two cluster solutions was perfect, we were confident that we could use our cluster solution as well. Also, when conducting MANOVAs with the new cluster solution as between subjects factor and the Big Five dimensions as dependent variables separately for the three separate waves, we found an almost identical pattern of these three personality types on the Big Five personality dimensions as depicted in Figure 1a-c. We can conclude that differences on the separate personality dimensions in comparison to previous studies, such as a higher level on agreeableness in undercontrollers, is not due to differences in initial cluster centres.

${ }^{2}$ We compared the stable and changing personality groups on gender, age, Big Five dimensions, depression and delinquency. The stable personality groups differed significantly from the changing personality groups on gender $\left(\chi^{2}(1)=7.77, p<0.01\right.$, adjusted residual $=2.8$; more girls than boys in the stable group; more boys than girls in the changing group), age $\left(\chi^{2}(1)=25.10, p<0.001\right.$, adj. res. $=5.0$; more early than middle adolescents in both the stable and changing group), agreeableness (within-subjects agreeableness x personality: $F(2,2166)=3.06$, $p<0.01$; higher increase in changing group than in stable group; between-subjects: $F(1,1083)=19.07$, $p<0.001$; stable $>$ changing), conscientiousness (between-subjects: $F(1,1086)=9.70, p<0.01$; stable $>$ chanchanging), openness to experience (between-subjects: $F(1,1076)=6.46, p<0.05$; stable $>$ changing) and delinquency (between-subjects: $F(1,1148)=4.2, p<0.05$; changing $>$ stable). Hence, the stable group differs from the changing group in several ways.
} 
decided to focus only on those adolescents who judged their personality consistently over time. $^{3}$

\section{Sample characteristics}

The sample consisted of 338 adolescents: 186 girls (55\%) and 152 boys (45\%). Two age groups were represented: 197 early adolescents $\left(58.3 \% ; \mathrm{M}_{\mathrm{ageT} 1}=12.37 ; \mathrm{SD}=0.55\right)$ and 141 middle adolescents $\left(41.7 \% ; \mathrm{M}_{\text {ageT1 }}=16.75 ; \mathrm{SD}=0.92\right)$.

Using the 338 adolescents that judged their personality type consistently over time, we found 114 stable resilients, 111 stable overcontrollers and 113 stable undercontrollers. The distribution of the genders differed within the personality types $\left(\chi^{2}(2)=24.54, p<0.001\right)$ : the percentage of girls was higher in the stable resilients $(62.3 \%$; adj. res. $=1.9)$ and stable overcontrollers (66.7\%; adj. res. $=3.0)$ than the percentage of boys, whereas the percentage of boys was higher in the stable undercontrollers $(63.7 \%$; adj. res. $=4.9)$. Additionally, the distribution of the age groups differed within the types $\left(\chi^{2}(2)=16.07\right.$, $p<0.001)$ : although the distribution of the younger and older adolescents were similar in the stable resilients (young: $50.9 \%$; adj. res. $=-2.0$ ) and stable overcontrollers (young: $50.5 \%$; adj. res. $=-2.0)$, there were more younger $(73.5 \%$; adj. res. $=4.0)$ than older adolescents in the stable undercontroller group.

\section{Strategy of Analyses}

To answer the first research question, we used two separate repeated measures analyses with Bonferroni's post hoc tests to investigate whether the adolescents as a whole group and the adolescents who judged their personality consistently over time showed stable differences in their mean level of depression and delinquency over the three waves. In both ANOVAs personality groups were entered as a between subjects factor; gender and age were included as covariates.

In order to answer the second research question, we first tested the co-occurrence in the adolescent sample and in the personality groups using bivariate correlations. Since we used an arbitrary selection of measurement waves, we averaged the correlations over the waves for each personality group and problem behaviour. Additionally, we tested whether the correlations differed between the groups by means of their confidence intervals. Then, we tested our two competing hypotheses in a set of multi-group analyses in Analysis of Moment Structures (AMOS 5; Arbuckle, 1995). For the multi-group analyses we compared

\footnotetext{
${ }^{3}$ We reran both models with five longitudinal personality groups $(N=568)$. According to Caspi and Silva (1995) and Herzberg and Roth (2006), two additional personality types may be present, namely a reserved personality group with both resilient and overcontroller attributes and a confident personality group with both resilient and undercontroller attributes (we are grateful to one of the reviewers to point this out to us). We constructed a reserved personality group by selecting those adolescents who switched between the resilient and overcontroller types $(n=92)$ and a confident personality group by selecting those adolescents who switched between the resilient and undercontroller types $(n=138)$ over the three waves. Since we found that the fit of the stability model did not differ significantly from the fit of the acting out/failure model $\left(\triangle \chi^{2}=29.47, \triangle d f=12, p>0.05\right)$, we chose the most parsimonious model, namely the stability model. When comparing the five personality groups on the stability of delinquency and the (mean) co-occurrence of depression and delinquency, we found the reserved personality group to be lower than the resilients but higher than the overcontrollers. Furthermore, we found the confident personality group to be lower than the resilients but higher than the undercontrollers. However, this pattern was not present on depression. Since the pattern of the changing personality groups was not clear on all stability or cooccurrence paths, we decided to restrict our study to the co-occurrence of depression and delinquency in the stable personality groups.
} 
various models: to test the acting out/failure hypothesis, we tested a multi-group model, in which acting out was present for overcontrollers and in which failure was present for undercontrollers. In this model, the cross paths from depression in wave 1 and 2 to delinquency in wave 2 and 3 , respectively were estimated for the stable overcontrollers, and set at zero for the stable resilients and stable undercontrollers. Additionally, the cross paths from delinquency in wave 1 and 2 to depression in wave 2 and 3, respectively were estimated for the stable undercontrollers and set at zero for the stable overcontrollers and stable resilients. To test the stability hypothesis, we tested a multi-group stability model for the three personality types in AMOS. In this model, we set the cross paths at zero for the three personality groups. Additional analyses were performed to test possible age group differences on the co-occurrence between depression and delinquency. For all abovementioned models, means and intercepts were estimated. The fit of the abovementioned models was assessed by several fit indices: $\chi^{2}$, CFI, RMSEA and AIC. The acting out/failure model and the stability model are compared with each other by using the $\chi^{2}$-comparison and the Akaike's Information Criterion (AIC). When comparing models by means of the AIC, the model with the lowest value is considered the best model (Kline, 1998).

\section{RESULTS}

The means and standard deviations of depression and delinquency for the total sample and the three stable personality groups are presented in Table 1.

For the first research question, the repeated measures ANOVA demonstrated that depression $(F(1,333)=775.92, p<0.001, \eta=0.70)$ changed significantly over the three waves: depression increased from wave 1 to wave 2, whereas it decreased from wave 2 to 3 . More importantly, the personality groups $(F(2,333)=41.60, p<0.001, \eta=0.20)$ showed significant effects on depression. Bonferroni's post hoc tests showed systematic differences on all three waves: the stable overcontrollers scored significantly higher on depression than both the stable resilients $(p<0.001)$ and the stable undercontrollers $(p<0.001)$. Additionally, the stable resilients scored significantly lower on depression than the stable undercontrollers $(p<0.05)$. Furthermore, gender $(F(1,333)=0.27, p>0.05, \eta=0.00)$ and age $(F(1,333)=0.47, p>0.05, \eta=0.00)$ did not show any significant effects. Finally, the ANOVAs demonstrated that the interactions of depression $\times$ gender, depression $\times$ age groups and depression $\times$ personality groups were not significant. This means that the change in depression did not differ significantly between the genders, age groups and personality groups over the three waves.

The second repeated measures ANOVA demonstrated that delinquency differed significantly over the waves $(F(1,333)=536.84, p<0.001, \eta=0.62)$ : delinquency decreased from wave 1 to wave 2 , whereas it increased from wave 2 to 3 . Furthermore, the personality groups showed significant effects $(F(2,333)=7.22, p<0.01, \eta=0.04)$. Bonferroni's post hoc tests showed that the stable undercontrollers scored significantly higher on delinquency than the stable resilients $(p<0.05)$ and the stable overcontrollers $(p<0.01)$ in all three waves. The stable resilients and the stable overcontrollers did not differ significantly from each other. Additionally, gender showed significant effects ( $F$ ( 1 , $333)=19.22, p<0.001, \eta=0.06$ ): boys were more delinquent than girls. However, no significant age group differences were found. Finally, the ANOVAs demonstrated that the interactions delinquency $\times$ gender, delinquency $\times$ age groups and delinquency $\times$ personpersonality groups were not significant. 
J. Akse et al.

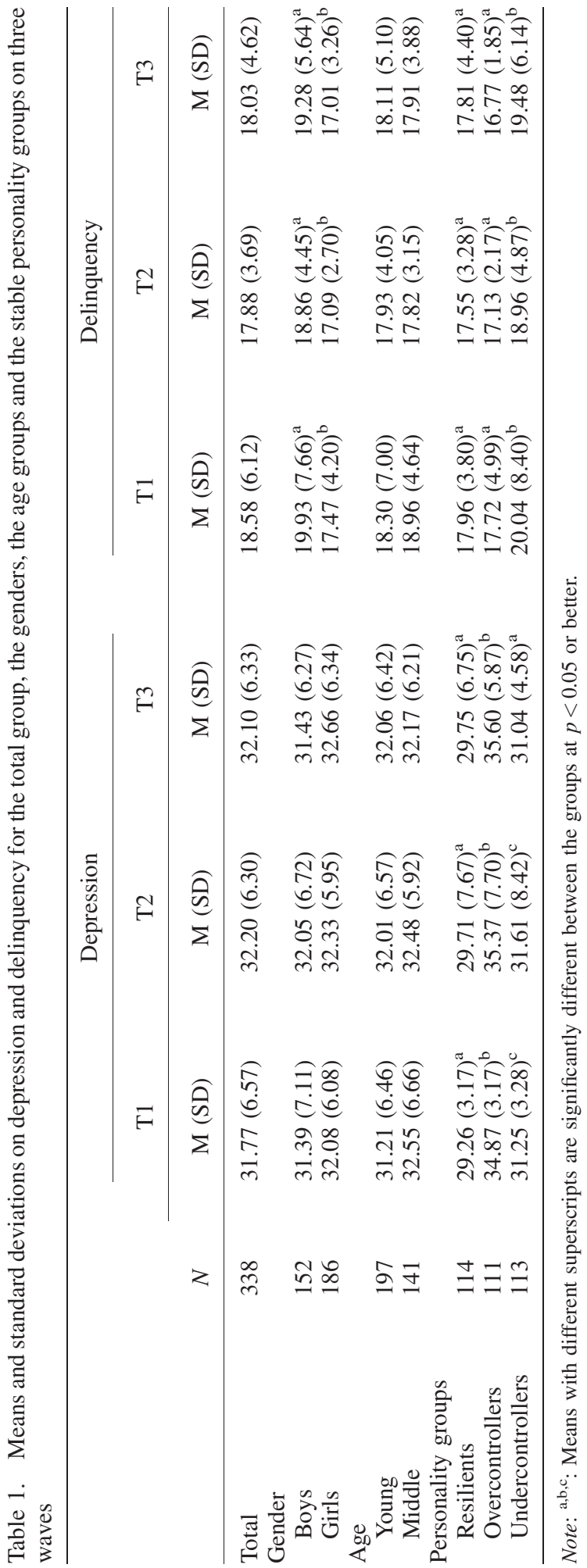


The second research question investigates whether the longitudinal co-occurrence of depression and delinquency is present in the adolescent sample and in the three personality types. Bivariate correlational analyses were conducted in the total adolescent sample $(N=338)$ and were found to be significant, which indicated that the co-occurrence between depression and delinquency was present for the total adolescent sample (T1: $r=0.13$, $p<0.05$; T2: $r=0.18, p<0.01$; T3: $r=-0.01, p>0.05$; Table 2). ${ }^{4}$ Additionally, we calculated the bivariate correlations for the three personality groups on three waves and

Table 2. Bivariate correlations between depression and delinquency in the total sample $(N=338)$ and in the three personality groups on the three waves

\begin{tabular}{|c|c|c|c|c|c|c|}
\hline & \multicolumn{3}{|c|}{ Depression } & \multicolumn{3}{|c|}{ Delinquency } \\
\hline & Wave 1 & Wave 2 & Wave 3 & Wave 1 & Wave 2 & Wave 3 \\
\hline \multicolumn{7}{|l|}{ Depression } \\
\hline \multicolumn{7}{|l|}{ Wave 1} \\
\hline Total & - & $0.52^{* *}$ & $0.47^{* *}$ & $0.13^{*}$ & 0.08 & 0.10 \\
\hline Resilients & - & $0.53^{* *}$ & $0.61^{* *}$ & $0.27^{* *}$ & $0.53^{* *}$ & 0.11 \\
\hline Overcontrollers & - & $0.51^{* *}$ & $0.41^{* *}$ & $0.19^{*}$ & 0.00 & -0.04 \\
\hline Undercontrollers & - & $0.34^{* *}$ & $0.29^{* *}$ & 0.13 & $0.19^{*}$ & $0.29^{*}$ \\
\hline \multicolumn{7}{|l|}{ Wave 2} \\
\hline Total & & - & $0.59^{* *}$ & $0.17^{* *}$ & $0.18^{* *}$ & 0.06 \\
\hline Resilients & & - & $0.57^{* *}$ & $0.41^{* *}$ & $0.55^{* *}$ & $0.47^{* *}$ \\
\hline Overcontrollers & & - & $0.66^{* *}$ & $0.21^{*}$ & 0.03 & -0.04 \\
\hline Undercontrollers & & - & 0.15 & 0.17 & $0.29^{* *}$ & 0.11 \\
\hline \multicolumn{7}{|l|}{ Wave 3} \\
\hline Total & & & - & 0.06 & 0.04 & -0.01 \\
\hline Resilients & & & - & $0.24^{* *}$ & $0.28^{* *}$ & $0.47^{* *}$ \\
\hline Overcontrollers & & & - & $0.20^{*}$ & 0.01 & 0.03 \\
\hline Undercontrollers & & & - & 0.02 & 0.12 & 0.02 \\
\hline \multicolumn{7}{|l|}{ Delinquency } \\
\hline \multicolumn{7}{|l|}{ Wave 1} \\
\hline Total & & & & - & $0.43^{* *}$ & $0.33^{* *}$ \\
\hline Resilients & & & & - & $0.65^{* *}$ & $0.57^{* *}$ \\
\hline Overcontrollers & & & & - & 0.15 & $0.19^{*}$ \\
\hline Undercontrollers & & & & - & $0.42^{* *}$ & $0.26^{* *}$ \\
\hline \multicolumn{7}{|l|}{ Wave 2} \\
\hline Total & & & & & - & $0.74^{* *}$ \\
\hline Resilients & & & & & - & $0.82^{* *}$ \\
\hline Overcontrollers & & & & & - & $0.65^{* *}$ \\
\hline Undercontrollers & & & & & - & $0.66^{* *}$ \\
\hline \multicolumn{7}{|l|}{ Wave 3} \\
\hline Total & & & & & & - \\
\hline Resilients & & & & & & - \\
\hline Overcontrollers & & & & & & - \\
\hline Undercontrollers & & & & & & - \\
\hline
\end{tabular}

${ }^{*} p<0.05$.

${ }^{* *} p<0.01$.

\footnotetext{
${ }^{4}$ When conducting bivariate correlational analyses on the total longitudinal adolescent sample $(N=940$; this sample contained adolescents who filled out the questionnaires about personality, depression and delinquency completely on the three waves and the sample consisted of adolescents who did or did not judge their personality type consistently over the three waves), we found the co-occurrence between depression and delinquency to be even more pronounced (T1: $r=0.14, p<0.01$; T2: $r=0.15, p<0.01$; T3: $r=0.08, p<0.01$ ).
} 
then averaged these correlations for each personality group (stable resilients: $r=0.43$; stable overcontrollers: $r=0.08$; stable undercontrollers: $r=0.15$ ). We found that the co-occurrence of depression and delinquency was significantly higher in stable resilients compared to stable overcontrollers $(z=2.81)$ and to stable undercontrollers $(z=2.29)$ over time.

We must point out that since it could be expected that overcontrollers show a uniformly high level of depression and undercontrollers show a uniformly high level of delinquency, the weaker correlation between depression and delinquency for these two groups might be explained by a restriction of range compared to the resilients (we are grateful to one of the reviewers who pointed this out to us). When using Gulliksen's adaptation of McNemar's formula to correct for possible range restrictions on the correlation between depression and delinquency and on their co-occurrence in the three personality groups (with the standard deviation of the resilient group as a reference point), we found that the correlations did not change a lot for depression or for the co-occurrence between depression and delinquency. ${ }^{5}$ However, the correlations on delinquency seemed to change. When averaging the corrected correlations of the personality groups over the three waves, the mean corrected correlation of the undercontrollers $(r=0.22)$ was lower compared to the uncorrected correlation $(r=0.45)$; the mean corrected value of the undercontrollers was even lower than the mean corrected value of the overcontrollers $(r=0.35)$. Additionally, the mean corrected correlation of the overcontrollers did not change compared to their mean uncorrected correlation $(r=0.33)$. This suggests that the findings of the stability of delinquency might be due to range restrictions; however, our results concerning the co-occurrence between depression and delinquency and concerning the stability of depression appear to be not due to the range restrictions. Furthermore, it appeared to be impossible to correct consistently for range restriction in AMOS (Bollen, 1989; Hox, personal communication, 26 January 2006).

Since we found clear personality group differences on the correlations between depression and delinquency over the waves, we could further test our hypotheses using structural equation modelling. First, we tested whether any co-occurrence was present for the total adolescent sample. Since we tested this in a model which consisted only of cooccurrent relations, the fit of the model was bad $\left(\chi^{2}(12)=613.45, p<0.001 ; \mathrm{CFI}=0.02\right.$, $\mathrm{RMSEA}=0.39, \mathrm{AIC}=643.45)$, which is due to the fact that the model is incomplete. However, the co-occurrence between depression and delinquency was present on wave 1 $(\beta=0.13, p<0.05)$ and $2(\beta=0.18, p<0.01)$, but not on wave $3(\beta=-0.01, p>0.05)$.

Second, in order to examine whether personality is a moderator in the longitudinal cooccurrence between depression and delinquency, we tested the acting out/failure model and the stability model in AMOS (Arbuckle, 1995; Figure 2a and b). Both the fit of the acting out/ failure model, in which acting out was present for the stable overcontrollers and in which failure was present for the stable undercontrollers (Figure $2 \mathrm{a} ; \chi^{2}(14)=32.72, p<0.01$; $\mathrm{CFI}=0.97, \mathrm{RMSEA}=0.06, \mathrm{AIC}=166.72$ ) and the fit of the stability model (Figure $2 \mathrm{~b}$; $\left.\chi^{2}(18)=37.05, p<0.01 ; \mathrm{CFI}=0.97, \mathrm{RMSEA}=0.06, \mathrm{AIC}=163.05\right)$ were acceptable. When comparing the fit of the acting out/failure model and the stability model, we did not find a significant difference $\left(\Delta \chi^{2}=4.33, \triangle d f=4, p>0.05\right)$, which implies that the most parsimonious model should be considered the best model; this is the stability model (Duncan, Duncan, Strycker, Li, \& Alpert, 1999). Also, the AIC was lowest in the stability model compared to the acting out/failure model. The stability model proved to describe the cooccurrence between depression and delinquency better than the acting out/failure model in

\footnotetext{
${ }^{5}$ We must point out that the way we corrected for range restriction could be somewhat unusual, since McNemar's formula is originally developed for meta-analyses.
} 
(a)

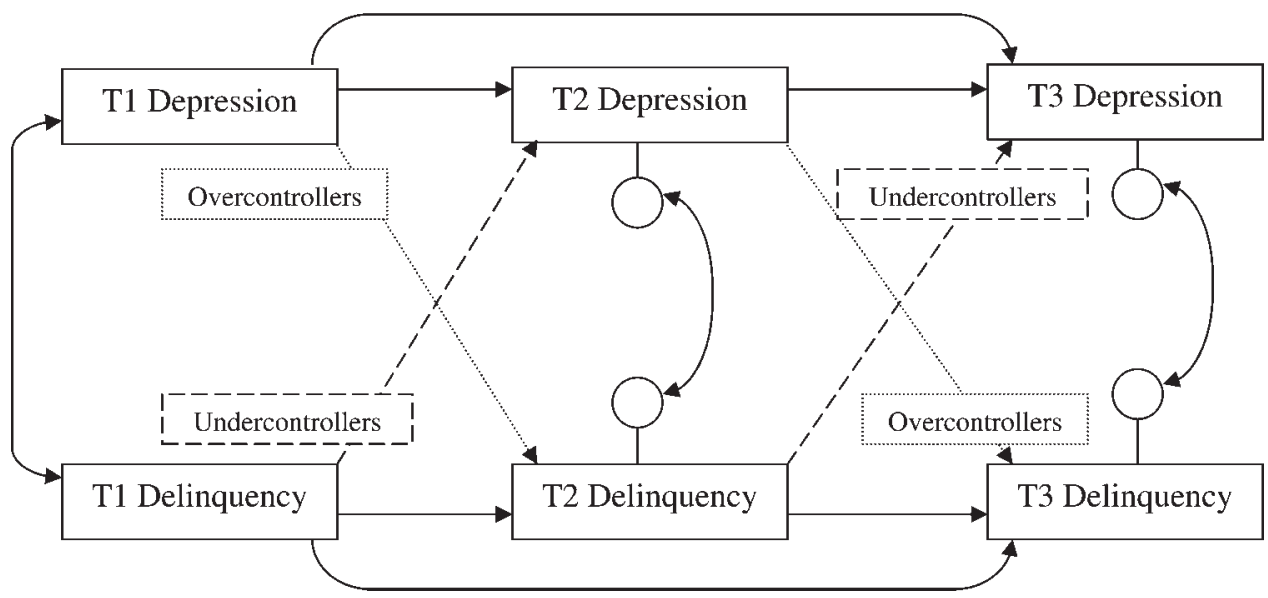

(b)

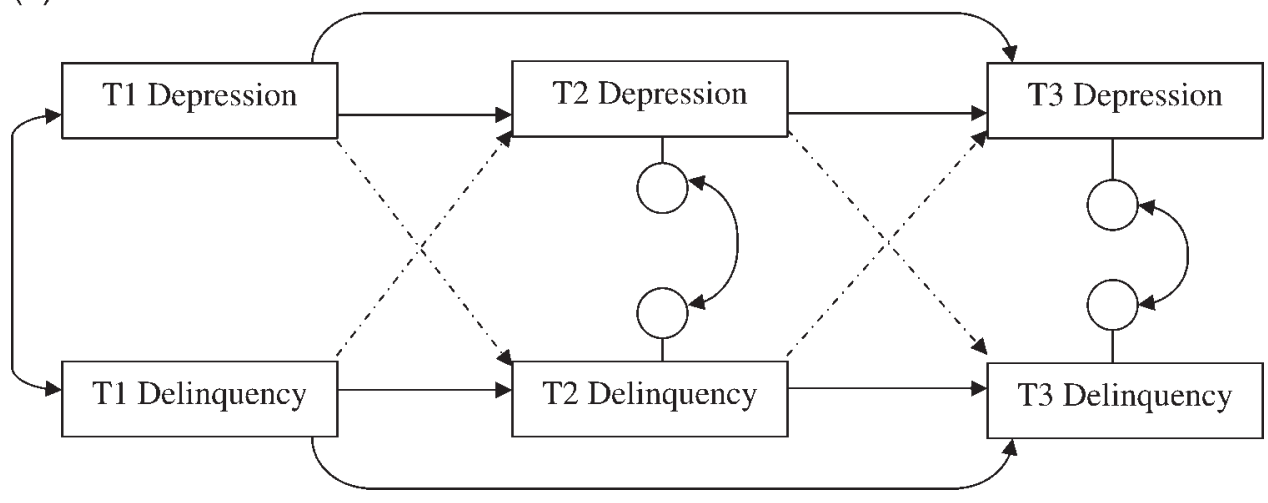

Figure 2. (a) A multi-group acting out/failure model. The cross paths from depression to delinquency (........) refer to acting out; the cross paths from delinquency to depression (----) refer to failure. (b) A multi-group stability model. The cross paths in the stability model are set at zero for the three personality groups.

these personality groups. Additionally, the acting out/failure model did not confirm the acting out/failure hypothesis: the paths from depression in wave 1 and 2 to delinquency in wave 2 and 3 , respectively were not significant for overcontrollers and the paths from delinquency in wave 1 and 2 to depression in wave 2 and 3 , respectively were not significant in undercontrollers. Table 3 summarises the findings of both multi-group models. ${ }^{6}$

\footnotetext{
${ }^{6}$ In order to elucidate what the stabilities between wave 1 and 3 mean, we analysed a stability model without depression and delinquency on wave $2(\chi 2(6)=13.22, p<0.05 ; \mathrm{CFI}=0.95$, RMSEA $=0.06, \mathrm{AIC}=85.22)$. The co-occurrence on wave 1 was the same for the model including and the model excluding depression and delinquency on wave 2 , whereas the correlated change on wave 3 (resilients: $r=0.16, p>0.05$; overcontrollers: $r=0.03, p>0.05$; undercontrollers: $r=-0.04, p>0.05$ ) differed from the model including wave 2. Furthermore, we found that the standardised regression weights between wave 1 and 3 were significantly higher for delinquency in the three personality groups (resilients: $r=0.62, p<0.001$; overcontrollers: $r=0.41, p<0.001$; undercontrollers: $r=0.30, p<0.001$ ) when delinquency on wave 2 was not included. This implies that delinquency on wave 2 perfectly mediates the association between delinquency on wave 1 and 3 . However, in depression no mediation was found (resilients: $r=0.56, p<0.001$; overcontrollers: $r=0.19, p<0.05$; undercontrollers: $r=0.26, p<0.01)$.
} 
J. Akse et al.

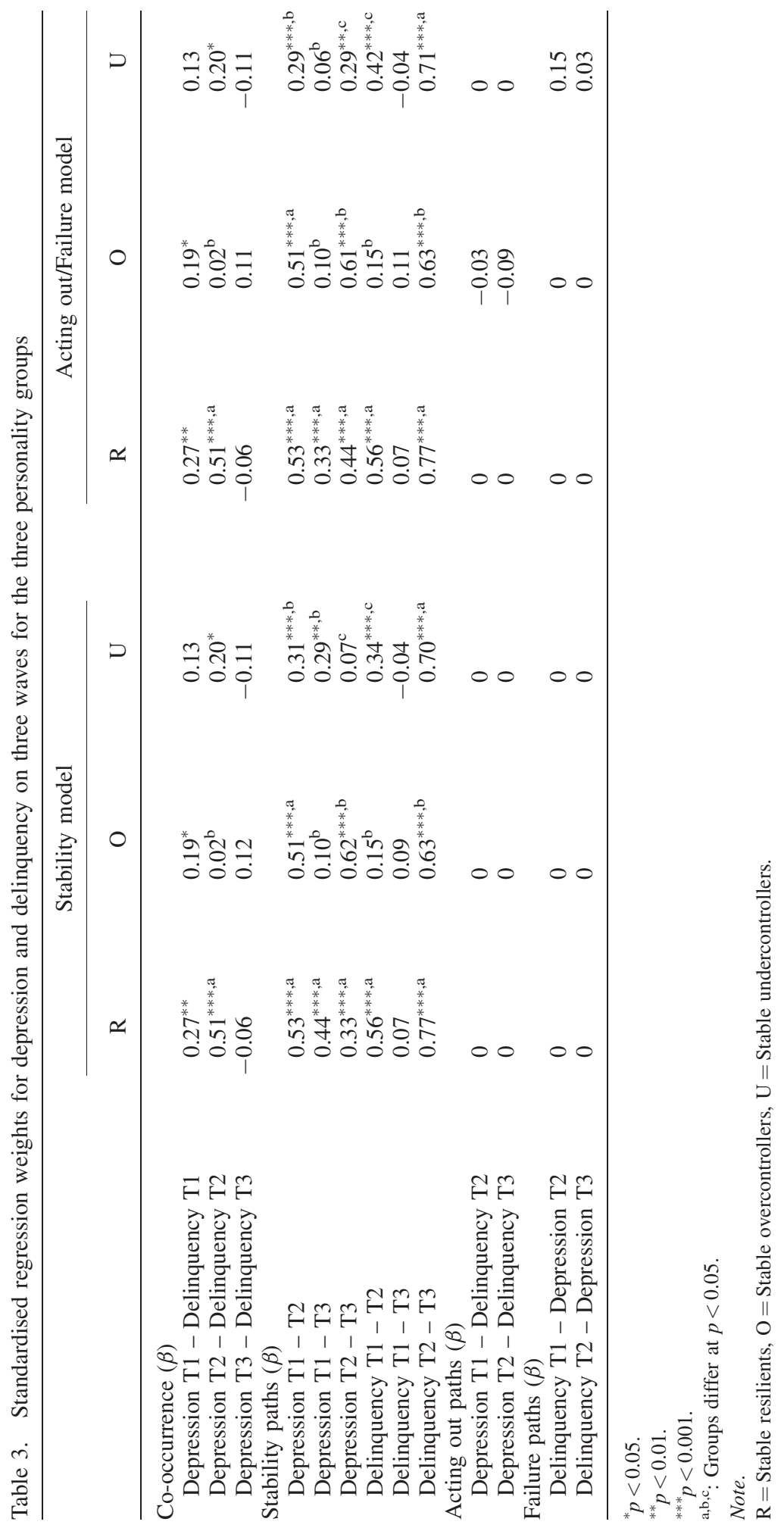


In inspecting the findings of the stability model, we first examined the parameter estimates between depression and delinquency on wave 1,2 and 3. We found that the standardised regression weights were significant for the stable resilients on wave 1 and 2, for the stable overcontrollers on wave 1 and for the stable undercontrollers on wave 2. This indicates that the co-occurrence on wave 1 , as well as the correlated change on wave 2 between depression and delinquency differs between the personality types; this was more present in the stable resilients than in the stable overcontrollers and undercontrollers.

In order to describe the findings on the stability paths of depression and delinquency, we calculated the total stability effects separately for both problem behaviours. A total effect is the sum of the direct and indirect effects. Direct effects in a path model depict causal effects that are presumed to flow from one variable to another. Indirect or mediator effects involve one or more intervening variables that transmit some of the causal effects of prior variables onto subsequent variables. An indirect effect is calculated as the product of the direct effects of which it consists (Kline, 1998). When examining significant group differences on these total stability effects we calculated a 95\% confidence interval for each group, separately for the problem behaviours.

The total stability effect of depression was highest in the stable resilients $(0.53 \times 0.33+0.44=0.61)$, followed by the stable overcontrollers $(0.51 \times 0.62+$ $0.10=0.42)$ and the stable undercontrollers $(0.31 \times 0.07+0.29=0.31)$. When testing for non-overlapping confidence intervals, we found that the total effect of depression was significantly higher in the stable resilients $($ lower $=0.48$, upper $=0.71)$ than in the stable undercontrollers (lower $=0.13$, upper $=0.47$ ). Both groups did not differ from the stable overcontrollers (lower $=0.25$, upper $=0.56$ ). We may conclude that the overall rank-order stability of depression was higher in the stable resilients than in the stable undercontrollers, with stable overcontrollers taking the intermediate position.

The total stability effect of delinquency was higher in the stable resilients $(0.56 \times 0.77+0.07=0.50)$ compared to total stability effect of the stable undercontrollers $(0.34 \times 0.70+-0.04=0.20)$ and the stable overcontrollers $(0.15 \times 0.63+0.09=0.18)$, although the confidence intervals overlapped slightly (resilients: lower $=0.35$, upper $=$ 0.63; overcontrollers: lower $=-0.01$, upper $=0.35$; undercontrollers: lower $=0.01$, upper $=0.37$. We may conclude that the stability of delinquency was higher in the stable resilients compared to the stable undercontrollers and the stable overcontrollers.

Additional analyses were performed to test possible personality $\times$ age group differences on the co-occurrence between depression and delinquency. We tested the stability model for six personality $\times$ age groups, namely the early $(n=58)$ and middle $(n=56)$ resilients, the early $(n=56)$ and middle $(n=55)$ overcontrollers and the early $(n=83)$ and middle $(n=30)$ undercontrollers. The fit of the six-group stability model was good $\left(\chi^{2}\right.$ $(36)=57.73, p<0.05 ; \mathrm{CFI}=0.97, \mathrm{RMSEA}=0.04, \mathrm{AIC}=309.73)$, which means that this stability model fit the data well. We calculated confidence intervals for the total stability effects to test for significant differences between the personality $\times$ age groups. In the early adolescents, the stability of delinquency in resilients $(\beta=0.57$, lower: 0.37 , upper: 0.72$)$ was significantly higher than the stability in overcontrollers $(\beta=0.03$, lower: -0.23 , upper: 0.29$)$ and undercontrollers $(\beta=0.16$, lower: -0.06 , upper: 0.36$)$. Furthermore, the stability of the middle overcontrollers $(\beta=0.54$, lower: 0.32 , upper: 0.70$)$ and middle undercontrollers $(\beta=0.70$, lower: 0.46 , upper: 0.85$)$ was significantly higher than the stability in the early overcontrollers and undercontrollers. The middle resilients $(\beta=0.58$, lower: 0.38, upper: 0.73) did not differ significantly from other groups. The stability on depression was not significantly different between the personality $\times$ age groups 


\section{J. Akse et al.}

(early resilients: $\beta=0.59$, lower: 0.39 , upper: 0.74 ; early overcontrollers: $\beta=0.32$, lower: 0.06, upper: 0.54 ; early undercontrollers: $\beta=0.35$, lower: 0.15 , upper: 0.53 ; middle resilients: $\beta=0.61$, lower: -0.11 , upper: 0.41 ; middle overcontrollers: $\beta=0.55$, lower: 0.33 , upper: 0.71 ; middle undercontrollers: $\beta=0.19$, lower: -0.18 , upper: 0.52 ). Finally, we may conclude that the rank-order stability of delinquency is significantly higher in middle adolescent overcontrollers and undercontrollers compared to early adolescent overcontrollers and undercontrollers, whereas the early and middle adolescent resilients displayed no significant age differences. We did not find significant personality $\times$ age group differences on the stability of depression.

\section{DISCUSSION}

The main goal of the current study was to examine whether personality moderated the longitudinal co-occurrence of depression and delinquency. Our first research question focused upon the validity of the three stable personality groups. We demonstrated that the longitudinal personality group differences were similar to previous cross-sectional studies (e.g. Dubas et al., 2002; Hart et al., 1997; Robins et al., 1996): the stable overcontrollers showed the highest mean level of depression (internalising) over time and the stable undercontrollers showed the highest mean level of delinquency (externalising) over time. The stable resilients exhibited the lowest mean level of both problem behaviours, representing the best psychosocial adjustment compared to the other two personality groups. Although not explicitly described in the study by van Aken and Dubas (2004), they found very similar personality group differences as found in the current study. Our findings indicate that when adolescents maintain their personality type membership over time, they also maintain the same level of internalising and externalising problem behaviours over time.

The second research question examined whether the longitudinal co-occurrence of depression and delinquency is present during adolescence and in the three personality groups. Furthermore, we examined whether personality type moderated the longitudinal co-occurrence between depression and delinquency. First, we demonstrated that the cooccurrence between depression and delinquency was present during adolescence, as was also found in a study by Overbeek et al. (2001). Second, we found that some co-occurrence was present in the personality groups. On the basis of these results we would suggest that the parallel development of depression and delinquency could be due to a third unknown factor. As hypothesized, the co-occurrence was significantly higher in the stable resilients compared to the stable overcontrollers and undercontrollers. This finding could be explained by the finding that ego-resiliency is related to stability in the overall environment (Asendorpf \& van Aken, 1991). Since resilients have a high level of ego-resiliency by definition, their overall environment could be more stable than the overall environment of overcontrollers and undercontrollers. Since the overall stability of the environment is thought to be higher in resilients in comparison to overcontrollers and undercontrollers, also the stability of depression and delinquency appears to be higher in resilients. Additionally, we also found the co-occurrence of depression and delinquency to be higher in resilients than in the overcontrollers and undercontrollers. This could be due to the fact that the amount of non-specific risk factors is different in resilients from the amount of nonspecific risk factors in the other personality types (Hart et al., 2003). 
Finally, we demonstrated that the longitudinal co-occurrence of depression and delinquency in the three personality groups was described more accurately in the stability model than in the acting out/failure model. We found that the co-occurrence of depression and delinquency differed between the personality groups and that it was more present in stable resilients than in stable overcontrollers and stable undercontrollers. Additionally, we found clear differences between the stable personality groups on the longitudinal stability of depression and delinquency. Based on the total effects, the stable resilients had a higher rank-order stability on depression compared to the stable undercontrollers. Furthermore, the stable resilients seemed to have a higher rank-order stability on delinquency compared to the stable overcontrollers and undercontrollers. Combining the high rank-order stability with the low mean levels (based on the repeated measures analyses) of the resilients on both problem behaviours, this means that these low mean levels are stable over time. Since we found that the resilients are not prone to developing a depressive mood and that they desist from delinquency, this is probably due to the fact that the resilients have the best resources to recover from negative events (Olsson, Bond, Burns, Vella-Brodrick, \& Sawyer, 2003) and that they have the ability to adapt to and to succeed in difficult contexts (Hart et al., 1997).

However, the stable overcontrollers and stable undercontrollers were found to have specific but different patterns of internalising and externalising problem behaviour. The stable overcontrollers demonstrated a moderate rank-order stability on depression and a low rank-order stability on delinquency (compared to the other groups), based on the calculation of the total effects of both problem behaviours. Combining these rank-order stabilities with the high mean level of depression and the low mean level of delinquency (based on the repeated measures analyses) in this group, this suggests a particular internalising pattern of problem behaviour. The undercontrollers demonstrated a moderate rank-order stability on delinquency and a low rank-order stability on depression (compared to the other groups), based on the calculation of the total effects of both problem behaviours. Combining these rank-order stabilities with the high mean level on delinquency and the low mean level of depression (based on the repeated measures analyses), this suggests a particular externalising pattern of problem behaviour. Hence, these two personality groups have the exact opposite longitudinal pattern of problem behaviour. This pattern of opposites could be explained as follows. Hart et al. (2005) found that although overcontrollers and undercontrollers are remarkably similar in terms of physiological and cognitive processes, which could be due to their low levels of ego-resiliency (Asendorpf \& van Aken, 1999; Robins et al., 1996), they differ radically at the behaviour level (Hart et al., 2005), which could be due to their markedly different levels of ego-control, namely high for overcontrollers and low for undercontrollers (Asendorpf \& van Aken, 1999; Robins et al., 1996). In this respect, ego-control may play an important role in explaining the opposite patterns of the problem behaviours over time, especially for the personality groups that do not respond flexibly to their environment, such as the overcontrollers and undercontrollers.

Since we found that the longitudinal co-occurrence of depression and delinquency in the three personality groups was described more accurately in the stability model than in the acting out/failure model, this implies that the associations between depression and delinquency maintain their stability over time in these personality groups. As Krueger (1999) and Krueger et al. (1998) point out, the co-occurrence of internalising and externalising problem behaviours may originate from general core psychopathological 
processes or non-specific risk factors (Fergusson et al., 1996; Wiesner, 2003). Possibly, the degree of ego-control is one of the common risk factors that could either lead to internalising or to externalising problem behaviour. Our results confirm this suggestion: we found that adolescents with an extreme high level of ego-control (overcontrollers) are prone to internalising problem behaviours, whereas the adolescents with an extreme low level of ego-control (undercontrollers) are prone to externalising problem behaviours.

Also, clear differences between the combined age $\times$ personality groups were found on the co-occurrence between depression and delinquency. We found that the rank-order stability of the stable resilients was the same in early and middle adolescents on depression and delinquency. However, the rank-order stability of the stable overcontrollers on depression seemed higher in the middle adolescents compared to the early adolescents, whereas the rank-order stability of the stable undercontrollers seemed lower in the middle adolescents than in the early adolescents. Furthermore, the rank-order stability of the undercontrollers on delinquency increased to an even higher level than the rank-order stability of the stable resilients. The rank-order stability of the stable overcontrollers also increased, but it did not reach the level of the stable resilients. These findings suggest that the rank-order stability increases in the problem behaviour to which a specific personality group is most prone. More specifically, the stable overcontrollers are prone to depression (Dubas et al., 2002; Robins et al., 1996): the middle adolescent overcontrollers seemed to demonstrate a higher rank-order stability on depression than early adolescent overcontrollers. Furthermore, the stable undercontrollers are more prone to delinquency (Dubas et al., 2002; Robins et al., 1996): the middle adolescent undercontrollers show a higher rank-order stability on delinquency than early adolescent undercontrollers. These findings are consistent with studies on trait consistency, which claim that traits become increasingly consistent with age (Roberts \& DelVecchio, 2000). Caution in interpreting these results should be warranted (Kline, 1998), since the personality $\times$ age groups are quite small. This issue should be tested in a larger sample.

Additionally, these findings demonstrate that the co-occurring problem behaviours do not constitute risk factors for each other in adolescents. Although the stable overcontrollers were prone to internalising problem behaviour (Dubas et al., 2002; Robins et al., 1996), they did not develop delinquency through acting out their depressed mood, which would be expected on the basis of Carlson and Cantwell's study (1980). Furthermore, although the stable undercontrollers were prone to externalising problem behaviour (Dubas et al., 2002; Robins et al., 1996), they did not develop depression through failure, which was hypothesized on the basis of the studies by Capaldi (1992) and by Beyers and Loeber (2003). An explanation for the different findings could be that the definitions of cooccurrence that were used in these studies differed from the definition we used in the current study. It should be noted that the aforementioned studies of longitudinal cooccurrence of internalising and externalising problem behaviour did not use personality type membership as a moderator. Our findings underline the importance of including personality in future studies of co-occurrence between internalising and externalising problem behaviour.

This is one of the first studies that examines the differences between the three personality groups in their rank-order stability of the co-occurrence between depression and delinquency. Changing in rank-order or the position relative to others in the group is unrelated to whether the group members show mean-level change (Asendorpf, 1992; Caspi, Roberts, \& Shiner, 2005; Roberts, Caspi, \& Moffitt, 2001; Roberts \& DelVecchio, 2000). The current study presents an important contribution to this issue, since the 
rank-order consistencies on internalising and externalising problem behaviours that we found were explicitly combined with the mean levels of the personality groups which we found in the repeated measures analyses.

In conclusion, we found the stability model to be the best fitting model to explain the co-occurrence of depression and delinquency in a general adolescent population, which is in line with the study by Overbeek et al. (2001). We extended Overbeek et al. (2001)'s study by employing personality type membership as a moderator in the co-occurrence of these problem behaviours, as was suggested by Wiesner (2003). Since we found clear personality group differences in the stability co-occurrence model, we concluded that personality type is an important moderator in the longitudinal co-occurrence of depression and delinquency.

\section{Limitations and Future Research}

In addition to the aforementioned findings, a few limitations of the present study need to be addressed. The first limitation is that our findings were solely based on adolescent selfreports, which could result in biased answers. Obviously, collecting data from multiple informants would improve our understanding of the associations between depression and delinquency during adolescence. However, since internalising behaviours might be more difficult to observe by others (Achenbach, McConaughy, \& Howell, 1987), we were specifically interested in the feelings and opinions of the adolescents themselves.

The second limitation of this study is that we did not examine possible moderator effects of gender in the structural equation modelling analyses. Although clear gender differences in the rank-order consistency of depression and delinquency were found (Wiesner, 2003) and since it was already found that personality type $\times$ gender proved to be an important moderator (Akse et al., 2004), we could not perform these multi-group analyses, since the personality $\times$ gender groups would be too small to get reliable results (Kline, 1998). This issue should be tested in a larger sample.

\section{REFERENCES}

Achenbach, T. M., McConaughy, S. H., \& Howell, C. T. (1987). Child/adolescent behavioral and emotional problems: Implication of cross-informant correlations for situational specificity. Psychological Bulletin, 101, 213-232.

Akse, J., Hale, W. W., III., Engels, R. C. M. E., Raaijmakers, Q. A. W., \& Meeus, W. H. J. (2004). Personality, perceived parental rejection and problem behaviour in adolescence. Social Psychiatry and Psychiatric Epidemiology, 39, 980-988.

American Psychological Association. (1994). Diagnostic and statistical manual of mental disorders (4th ed.). Washington, D.C: American Psychological Association.

Angold, A., Costello, E. J., \& Erkanli, A. (1999). Comorbidity. Journal of Child Psychology and Psychiatry, 40, 57-87.

Arbuckle, J. L. (1995). AMOS 4.0. Chicago: SmallWaters Corporation.

Asendorpf, J. B. (1992). Continuity and stability of personality traits and personality patterns. In J. B. Asendorpf, \& J. Valsiner (Eds.), Stability and change in development (pp. 116-154). Newbury Park: Sage Publications.

Asendorpf, J. B. (2006). Typeness of personality profiles: A continuous person-centred approach to personality data. European Journal of Personality, 20, 83-106. 
Asendorpf, J. B., \& van Aken, M. A. G. (1991). Correlates of the temporal consistency of personality patterns in childhood. Journal of Personality, 59, 689-703.

Asendorpf, J. B., \& van Aken, M. A. G. (1999). Resilient, overcontrolled, and undercontrolled personality prototypes in childhood: Replicability, predictive power, and the trait-type issue. Journal of Personality and Social Psychology, 77, 815-832.

Baerveldt, C. (2000). Pupil's networks in high schools: Network sampling, program and some results from a theory-oriented research project on petty crime of pupils. Paper presented at the 2nd International Network Sampling Workshop, Maastricht, The Netherlands (March 2-4).

Baerveldt, C., van Rossem, R., \& Vermande, M. (2003). Pupils' delinquency and their social networks: A test of some network assumptions of the ability and inability models of delinquency. The Netherlands Journal of Social Sciences, 39, 107-125.

Ben-Amos, B. (1992). Depression and conduct disorders in children and adolescents: A review of the literature. Bulletin of the Menninger Clinic, 56, 188-224.

Beyers, J. M., \& Loeber, R. (2003). Untangling developmental relations between depressed mood and delinquency in male adolescents. Journal of Abnormal Child Psychology, 31, 247-266.

Block, J. H., \& Block, J. (1980). The role of ego-control and ego-resiliency in the organization of behavior. In W. A. Collins (Ed.), Development of cognition, affect, and social relations (Vol. 13, p. 294). Hillsdale: Lawrence Erlbaum Associates.

Bollen, K. A. (1989). Structural equations with latent variables. New York: John Wiley \& Sons.

Burke, J. D., Loeber, R., Lahey, B. B., \& Rathouz, P. J. (2005). Developmental transitions among affective and behavioral disorders in adolescent boys. Journal of Child Psychology and Psychiatry, 46, 1200-1210.

Capaldi, D. M. (1992). Co-occurrence of conduct problems and depressive symptoms in early adolescent boys: II. A 2-year follow-up at grade 8. Development and Psychopathology, 4, 125-144.

Carlson, G. A., \& Cantwell, D. P. (1980). Unmasking masked depression in children and adolescents. American Journal of Psychiatry, 137, 445-449.

Caspi, A., Roberts, B. W., \& Shiner, R. (2005). Personality development: Stability and change. Annual Reviews of Psychology, 56, 453-484.

Caspi, A., \& Silva, P. A. (1995). Temperamental qualities at age three predict personality traits in young adulthood: Longitudinal evidence from a birth cohort. Child Development, 66, 486-498.

Cohen, J. A. (1960). A coefficient of agreement for nominal scales. Educational and Psychological Measurement, 20, 37-46.

Craighead, W. E., Smucker, M. R., Craighead, L. W., \& Ilardi, S. S. (1998). Factor analysis of the Children's Depression Inventory in a community sample. Psychological Assessment, 10, 156-165.

Digman, J. M. (1990). Personality structure: Emergence of the five-factor model. Annual Review of Psychology, 41, 417-440.

Dubas, J. S., Gerris, J. R. M., Janssens, J. M. A. M., \& Vermulst, A. A. (2002). Personality types of adolescents: Concurrent correlates, antecedents, and type $\times$ parenting interactions. Journal of Adolescence, 25, 79-92.

Duncan, T. E., Duncan, S. C., Strycker, L. A., Li, F., \& Alpert, A. (1999). An introduction to latent variable growth curve modeling: Concepts, issues, and applications. Mahwah, NJ: Lawrence Erlbaum Associates.

Fergusson, D. M., Lynskey, M. T., \& Horwood, L. J. (1996). Origins of comorbidity between conduct and affective disorders. Journal of the American Academy of Child and Adolescent Psychiatry, 35, $451-460$.

Gerris, J. R. M., Houtmans, M. J. M., Kwaaitaal-Roosen, E. M. G., Schipper, J. C., Vermulst, A. A., \& Janssens, J. M. A. M. (1998). Parents, adolescents and young adults in Dutch families: A longitudinal study. Nijmegen: Institute of Family Studies University of Nijmegen.

Gold, M., Mattlin, M., \& Osgood, D. W. (1989). Background characteristics and responses to treatment of two types of institutionalized delinquent boys. Criminal Justice and Behaviour, $16,5-33$.

Goldberg, L. R. (1992). The development of markers for the Big-Five factor structure. Psychological Assessment, 4, 26-42.

Hart, D., Atkins, R., \& Fegley, S. G. (2003). Stability and change in personality types. Monographs of the Society for Research in Child Development, 68, 62-73. 
Hart, D., Burock, D., London, B., Atkins, R., \& Bonilla-Santiago, G. (2005). The relation of personality types to physiological, behavioural, and cognitive processes. European Journal of Personality, 19, 391-407.

Hart, D., Hofmann, V., Edelstein, W., \& Keller, M. (1997). The relation of childhood personality types to adolescent behavior and development: A longitudinal study of Icelandic children. Developmental Psychology, 33, 195-205.

Herzberg, P. Y., \& Roth, M. (2006). Beyond resilients, undercontrollers, and overcontrollers?: An extension of personality prototype research. European Journal of Personality, 20, 5-28.

Hodges, K. (1990). Depression and anxiety in children: A comparison of self-report questionnaires to clinical interview. Psychological Assessment, 2, 376-381.

Keiley, M. K., Lofthouse, N., Bates, J. E., Dodge, K. A., \& Pettit, G. S. (2003). Differential risks of covarying and pure components in mother and teacher reports of externalizing and internalizing behavior across ages 5 to 14. Journal of Abnormal Child Psychology, 31, 267-283.

Kline, R. B. (1998). Principles and practice of Structural Equation Modelling. New York: The Guilford Press.

Kovacs, M. (1985). The Children's Depression Inventory (CDI). Psychopharmacology Bulletin, 21, 995-998.

Krueger, R. F. (1999). The structure of common mental disorders. Archives of General Psychiatry, 56, 921-926.

Krueger, R. F. (2005). Continuity of axis I and II: Toward a unified model of personality, personality disorders, and clinical disorders. Journal of Personality Disorders, 19, 233-261.

Krueger, R. F., Caspi, A., \& Moffitt, T. E. (2000). Epidemiological personology: The unifying role of personality in population-based research on problem behaviors. Journal of Personality, 68, $967-$ 998.

Krueger, R. F., Caspi, A., Moffitt, T. E., \& Silva, P. A. (1998). The structure and stability of common mental disorders (DSM-III-R): A longitudinal-epidemiological study. Journal of Abnormal Psychology, 107, 216-227.

Krueger, R. F., \& Markon, K. E. (2006). Reinterpreting comorbidity: A model-based approach to understanding and classifying psychopathology. Annual Reviews of Clinical Psychology, 2, 2.1-2.23.

McCrae, R. R., \& Costa, P. T. J. (1995). Trait explanations in personality psychology. European Journal of Personality, 9, 231-252.

Meeus, W., Akse, J., Branje, S., Crommelin, P., de Goede,I., Delsing, M., Engels, R., Finkenauer, R., Hale, W. W., III., Keijsers, L., Raaijmakers, Q., Selfhout, M., Spruijt, E., ter Bogt, T., van Doorn, M., \& van der Valk, I. (2002). CONAMORE: CONflict And Management Of RElationships, (Unpublished raw data).

Meller, W. H., \& Borchardt, C. M. (1996). Comorbidity of major depression and conduct disorder. Journal of Affective Disorders, 39, 123-126.

Olsson, C. A., Bond, L., Burns, J. M., Vella-Brodrick, D. A. V., \& Sawyer, S. M. (2003). Adolescent resilience: A concept analysis. Journal of Adolescence, 26, 1-11.

Overbeek, G. J., Vollebergh, W., Meeus, W., Engels, R., \& Luijpers, E. (2001). Course, cooccurrence, and longitudinal associations of emotional disturbances and delinquency from adolescence to young adulthood: A six-year three-wave study. Journal of Youth and Adolescence, 30, 401-426.

Roberts, B. W., Caspi, A., \& Moffitt, T. E. (2001). The kids are alright: Growth and stability in personality development from adolescence to adulthood. Journal of Personality and Social Psychology, 81, 670-683.

Roberts, B. W., \& DelVecchio, W. F. (2000). The rank-order consistency of personality traits from childhood to old age: A quantitative review of longitudinal studies. Psychological Bulletin, 126, $3-$ 25.

Robins, R. W., John, O. P., Caspi, A., Moffitt, T. E., \& Stouthamer-Loeber, M. (1996). Resilient, overcontrolled, and undercontrolled boys: Three replicable personality types. Journal of Personality and Social Psychology, 70, 157-171.

Smith, D. R., \& Snell, W. E. Jr. (1996). Goldberg's bipolar measure of the Big Five personality dimensions: Reliability and validity. European Journal of Personality, 10, 283-299. 
van Aken, M. A. G., \& Dubas, J. (2000). Personality types and perceived support in adolescence: Main effects and person-support transactions. Paper presented at the First Expert Workshop on Personality Psychology. Ghent, Belgium.

van Aken, M. A. G., \& Dubas, J. (2004). Personality type, social relationships, and problem behavior in adolescence. European Journal of Developmental Psychology, 1, 331-348.

van Leeuwen, K. G., Mervielde,I., Braet, C., \& Bosmans, G. (2004). Child personality and problem behavior as moderators of problem behavior: Variable- and person-centered approaches. Developmental Psychology, 40, 1028-1046.

Wiesner, M. (2003). The longitudinal latent variable analysis of reciprocal relations between depressive symptoms and delinquency during adolescence. Journal of Abnormal Psychology, $112,633-645$.

Youngstrom, E., Findling, E. A., \& Calabrese, J. R. (2003). Who are the comorbid adolescents? Agreement between psychiatric diagnosis, youth, parent, and teacher report. Journal of Abnormal Child Psychology, 31, 231-245. 